\title{
Unmanned Monitoring System using Motion Detection and Ultrasonic Signals
}

\author{
M. S. Islam, J. C. Lee and U. P. Chong*
}

\begin{abstract}
This paper presents unmanned monitoring system. This system based on the motion sensors, ultrasonic sensor, and camera has been constructed, which is considerably inexpensive compared with the conventional 3D motion analysis system based on high-speed camera. This unmanned monitoring system which can detect the targets in the concerning region using the motion detector and then capture the image of target using camera. Moreover, this proposed approach calculates the distance of targets using cross correlation of received signal from the ultrasonic sensor. The experimental result shows the reliability of the system.
\end{abstract}

Keywords - Unmanned monitoring, Motion detection, Ultrasonic sensor, Mobile monitoring, Wireless communication.

\section{Introduction}

The rapid development of communication and network technology has speeded up the digital control system to replace the pace of analog control system; camera based monitoring has become an inevitable trend in monitoring technology. Over the last decade, many camera based monitoring systems have been extensively developed and implemented for numerous applications. The increasing requirements of the markets, which include banks, companies and other important institutes [1] are established on three main concepts; low power, less cost and high efficiency.

An increasing amount of attention has been paid to improve reliability, availability, and safety in most autonomous control systems such as remote control station. For instance, the control systems in the field of aerospace, electric power and automobiles become more complicated, more rigorous research for Fault Detection and Diagnosis (FDD). For these reasons, a fault tolerant state estimation and diagnosis method with high reliability are developed for the reliable operations of remote control station in combat fields.

The authors in [2] introduce a potential technique for monitoring the indoor environment. A web-based building environmental monitoring system is proposed in [3], based on wireless communication scheme.

\footnotetext{
This work (Grants No. C0183423) was supported by Business for Cooperative R\&D between Industry, Academy, and Research Institute funded Korea Small and Medium Business Administration in 2014.

M. S. Islam, J. C. Lee and U. P. Chong*

University of Ulsan, School of Electrical Engineering

University of Ulsan, Whale Research Institute*

Ulsan, South Korea
}

In terms of performance metrics in [4], some applications have been discussed in the building monitoring system. Moreover, a wireless network based monitoring system was deployed in a number of residential and commercial buildings in $[5,6]$. For these approaches a human operator is required to monitor the camera images at all times when security is to be maintained, visually scanning the images to determine whether or not suspicious activity is taking place. This is a monotonous task which may lead to operator fatigue and inevitably results in errors [7]. Currently, the data link between cameras onboard an unmanned aerial vehicle and the monitoring terminal [8] and new technological solution for the ocean observation using an unmanned surface vehicle [9] have been researched. Kinematic and dynamic analyses have been done and the mathematical model is developed for the marine platform of the surface vehicle [9].

The objective of this paper is to develop an unmanned monitoring system achieving the requirement of a real time security system, which is expected to calculate the distance of object at the same time. The basic concept of the proposed method is to detect the targets in the region using the motion detector and then capture the images of target using camera. Moreover, this proposed approach calculates the distance of targets using ultrasonic sensor. This approach is considerably inexpensive compared with the conventional 3D motion analysis system based on high-speed camera.

The rest of the paper is organized as follows: Section 2 describes the design of unmanned monitoring system including motion detection, image captures and ultrasonic signal processing. Section 3 shows the implementation results of hardware and software. Finally, the conclusions are presented in Section 4.

\section{Design of unmanned monitoring system}

Flow chart of overall unmanned monitoring system is presented in figure 1. This unmanned monitoring system which can detect the targets in the concerning region using the motion detector and then capture the image of target using camera. There are four detection regions (I, II, III, IV) which detect the objects from two motion sensors in the figure 1 . When the control unit receives detection signal from motion detectors it sends signal to servo motor. Then server motor moves toward the detected region and the camera takes pictures and ultrasonic sensor sends/receives signals from/to 
target at same time. Then control unit sends the images to the server computer through wireless communication. The server computer calculates the distance between sensors and target using the ultrasonic signals, and sends information to mobile devices.

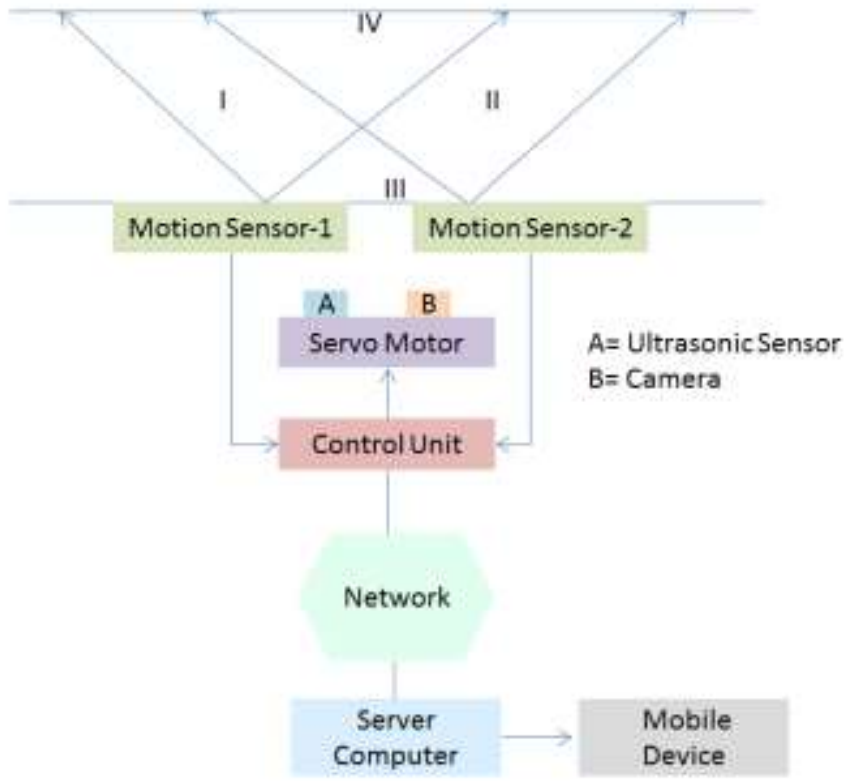

Figure 1: Overall block diagram for unmanned monitoring system

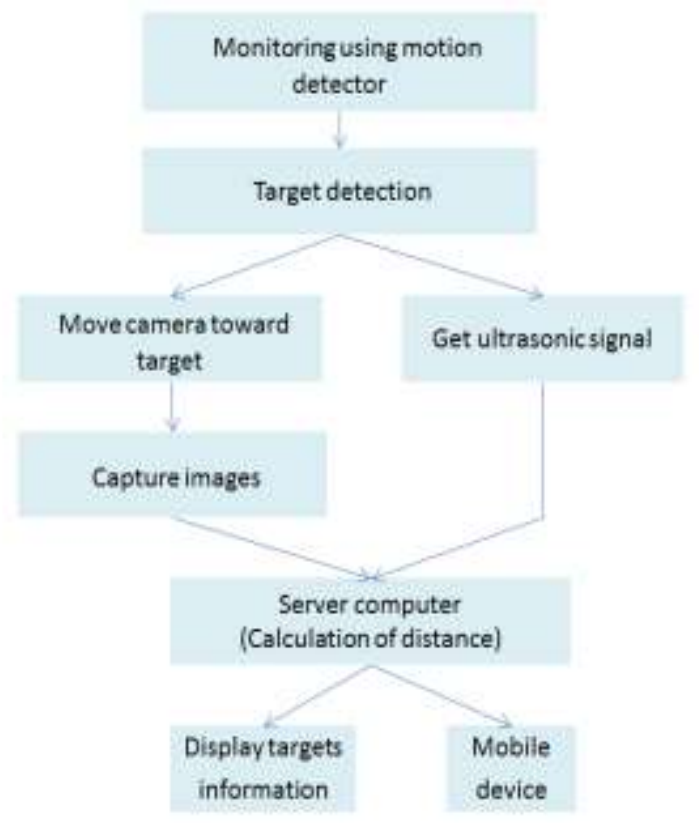

Figure 2: Flowchart of unmanned monitoring system
Flow chart of overall unmanned monitoring system has been presented in figure 2. This unmanned monitoring system which can detect the targets in the region using the motion detector and then capture the images of targets using digital camera. Moreover, this proposed approach calculates the distance of targets using ultrasonic sensor with cross correlation. It will be discussed later.

\section{A. Motion Detection and Image Capture}

The main disadvantages of existing monitoring systems are to continuously capture or record the video. So the system consumes huge power and need large memory to store the database of the captured image or video. To overcome these disadvantages we proposed the new system working only motion detection occurred.

Motion detection system consists of motion detector, control board, servo motor, digital camera, and wireless communication system as shown figure 3. There are two motion detector in our system. The motion sensor-1 keeps monitoring left detection region(region I) and motion sensor- 2 keeps monitoring right detection region(region II) as shown figure 1. The mid region(region IV) is detected when both motion detectors are activated. Detection region III is not necessarily for detection because region IV already covers it. When the motion detectors detect the target in any of four region, then control unit sends signal to servo motor to move toward that detected region. Digital camera(IP camera) takes pictures toward the target and wireless mobile system transfers it to server computer system.

We analyzed camera image transmission format with image transmission protocol to get image from IP camera. VSTARCAM-100S uses JPEG format for the images. The firmware in the IP camera controls the CGI and accesses to URL. The camera transmits the images through http protocol to the client when URL is accessed.

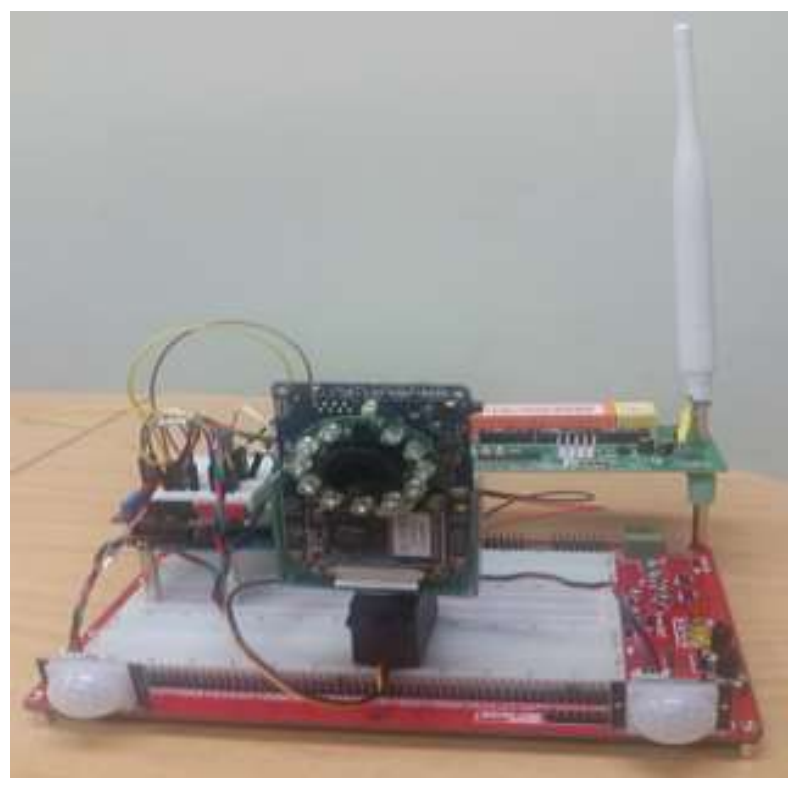

Figure 3: Motion detection and image capture system 


\section{B. Distance Calculation using Ultrasonic Signals}

Ultrasonic distance detection is based on the time of flight of an ultrasonic pulse. An ultrasonic pulse will be partially reflected from the object. The time difference between transmitting a pulse and receiving the reflected signal is a measure for the distance between the transmitter and the object. We use the cross correlation to find the time difference. Correlation is the process to determine degree of 'fit' between two waveforms and to determine the time at which the maximum correlation coefficient or "best fit" occurs. For this system, we correlate between the transmitted signal and the received signal, then we get the time difference between transmitted and received signal. The figure 4 shows experimental setup of ultrasonic sensors.

In this section we will drive the equation of the crosscorrelation for distance calculation. If we consider $\mathrm{x}(\mathrm{n})$ as the transmitted signal from the ultrasonic sensor, then the returned signal, $r(n)$ may be modeled as:

$$
r(n)=\alpha x(n-D)+w(n)
$$

Where $w(n)$ is assumed to be the additive noise during the transmission, $\alpha$ is the attenuation factor, D is the delay which is the time taken for the signal to travel from the transmitter to the target and back to the receiver.

Now the auto-correlation of the transmitted signal $\mathrm{x}(\mathrm{n})$ with itself (constant shift $l$ ) be [10]

$$
C_{x x}(l)=\sum_{n=-\infty}^{n=+\infty} x(n) x(n-l)
$$

The maximum value of the auto-correlation will be at the delay time $l$. The cross-correlation between the transmitted signal, $x(n)$ and the received signal, $r(n)$ be [10]

$$
\begin{aligned}
C_{x r}(l) & =\sum_{n=-\infty}^{n=+\infty} r(n) x(n+l) \\
& =\sum_{n=-\infty}^{n=+\infty}[\alpha x(n-D)+w(n)][x(n+l)] \\
& =\sum\{\alpha x(n-D) x(n+l)+w(n) x(n+l)\}
\end{aligned}
$$

Now using Equation (2) and (3)

$$
C_{x r}(l)=\alpha C_{x x}(l-D)+C_{w x}(l)
$$

Since the noise signal $\mathrm{w}(\mathrm{n})$ and the transmitted signal, $\mathrm{x}(\mathrm{n})$ are uncorrelated then, $\mathrm{C}_{\mathrm{wx}}(l)=0$. Therefore equation (4) will be

$$
C_{x r}(l)=\alpha C_{x x}(l-D)
$$

Comparing the equation (5) with equation (2), the maximum value of the cross-correlation will occur at $l=\mathrm{D}$, which is our interest in cross-correlation from which we can detect our target.

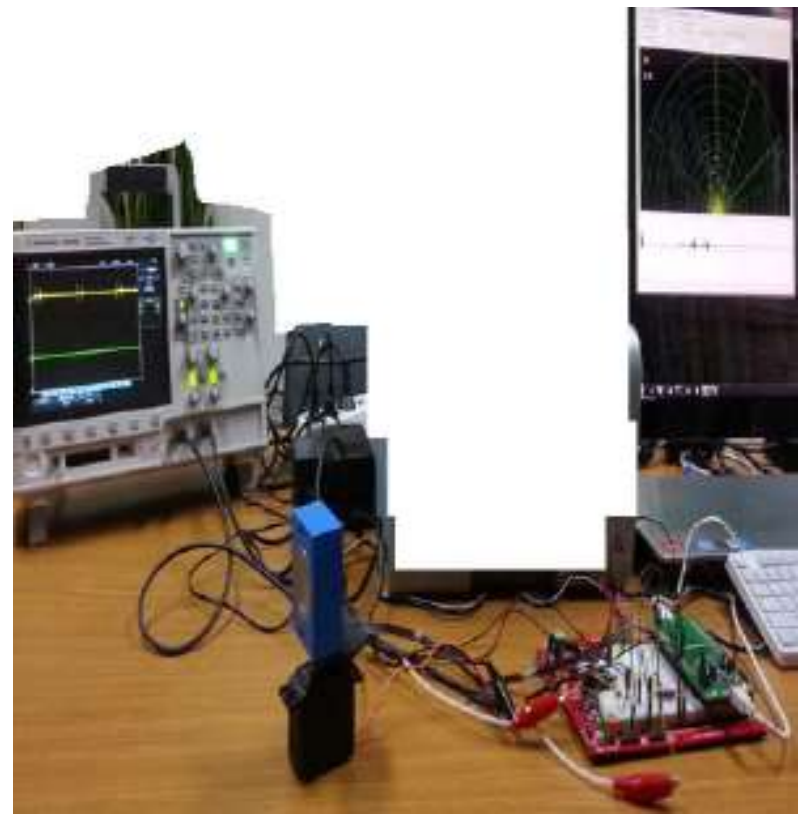

Figure 4. Experimental setup of ultrasonic sensors

\section{Implementation and Results}

\section{A. Motion Detection and Image Capture}

The hardware implementation for motion detection and image capture is shown in figure 3. The operating procedure is as follows.

1. Motion detectors keep monitoring the detection region

2. Motion detectors recognize the region when motion occurred and send detection signal to control board

3. Control part activates the servo motor

4. The motor with camera turns to detected area

5. Camera takes pictures and sends information to sever computer through wireless communication

6. The computer displays the target information and transfers information to mobile device

\section{B. Distance Calculation of Targets}

Experimental set up to get distance information is shown figure 4. Figure 5 represents the experimental ultrasonic 
transmitted and received signal. Experiments are carried out, in which tranmitted signal contains 16 pulses with frequency, $50 \mathrm{kHz}$ and sampling frequency, $500 \mathrm{kHz}$. The cross correlation between transmitted signal and received signal is shown in figure 6. Using the correlation coefficients from equation (5), target distance is calculated. This distance from the target can be calculated by the figure 6 .

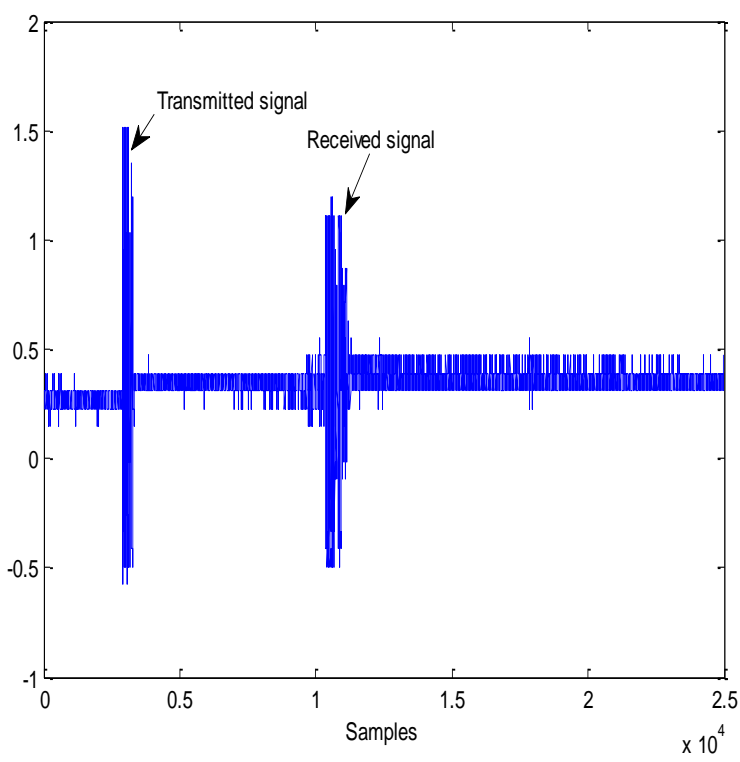

Figure 5: Ultrasonic transmitted signal and received signal

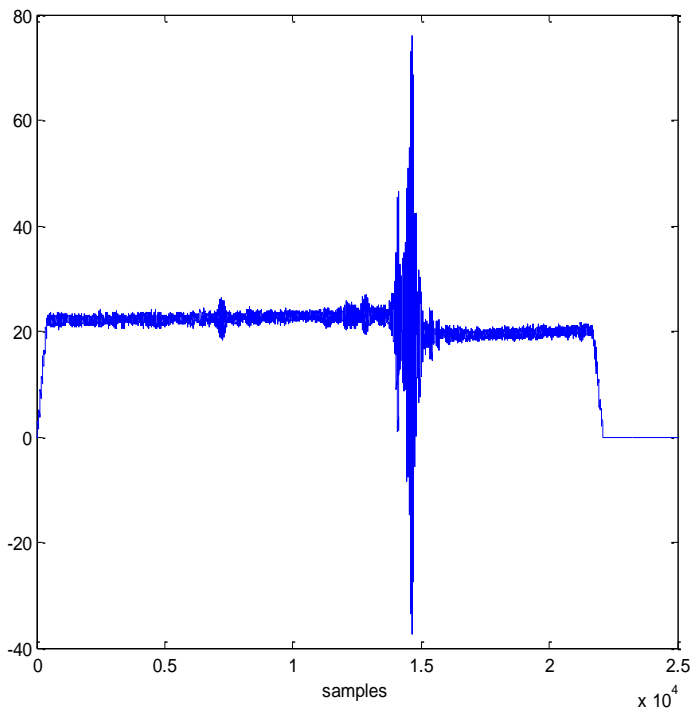

Figure 6: Results of cross correlation

\section{Display of Radar Format Indicator}

Display of PC based radar format consists of control board and communication, connection to control module, azimuth of control information part, GUI interface. Figure 7 shows the output display of PC based radar format from the target indication of the received ultrasonic signals.

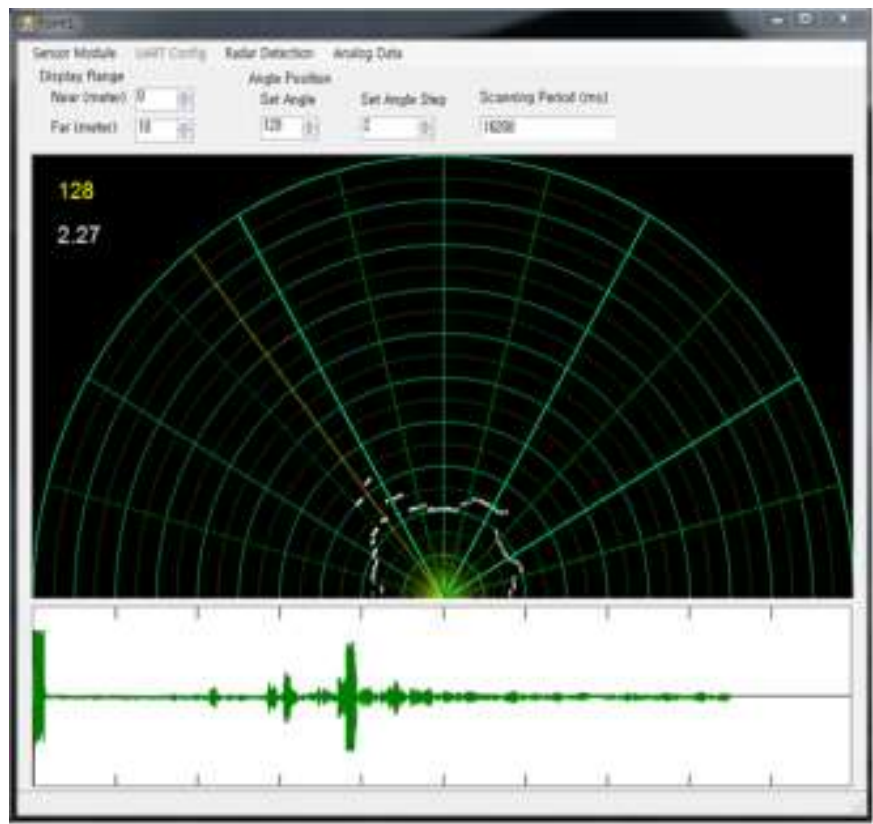

Figure 7: GUI and Display of PC based Target Area

\section{Conclusions}

The proposed unmanned monitoring system can detect the targets in the concerning region using the motion sensors. This system can capture the target image by camera and also detect the target distance by ultrasonic sensor. Hardware implementation is completed and tested with software we designed. The results showed that the developed system works properly and can apply to the actual fields. We need the system upgrade for reliability and security purpose in the future.

\section{References}

[1] V. Boonsawat, J. Ekchamanonta, K. Bumrungkhet, and S. Kittipiyakul, "Xbee wireless sensor networks for temperature monitoring," ECTICARD 2010, pp. 10-14 .

[2] Q. Dong, "Design of Building Monitoring Systems Based on Wireless Sensor Networks,". Journal of Wireless Sensor Network, Vol. 2 No. 9, p. 703-709, 2010

[3] Won-Suk Jang, William M. Healy, Miroslaw J. Skibniewski, "Wireless sensor networks as a part of a web-based building environmental monitoring system,"Automation in Construction Volume 17, Issue 6, August2008, Pages 729-736

[4] W. S. Jang and W. M. Healy, "Wireless Sensor Network Performance Metrics for Building Applications," Energy and Buildings, Vol. 42, No. 6, 20IO, pp. 862-868 
Proc. of the Second Intl. Conf. on Advances in Information Processing and Communication Technology - IPCT 2015

Copyright $(\odot$ Institute of Research Engineers and Doctors, USA .All rights reserved.

ISBN: 978-1-63248-044-6 doi: 10.15224/ 978-1-63248-044-6-71

[5] D. Tessa, G. Elena and B. James, "Wireless Sensor Networks to Enable the Passive House- Deployment Experiences," Smart Sensing and Context, Berlin HeIdelberg: Springer, 2009, pp. 177-192

[6] Amit Verma, "A Multi Layer Bank Security System," 2013 International Conference on Green Computing, Communication and Conservation of Energy (ICGCE), Chennai, p. 914-917.

[7] J. A. Freer, B. J. Beggs, H. L. Femandez-Canque, F. Chevriert, A. Goryashkot, "Moving Object Surveillance and Analysis for Camera Based Security Systems", International carnahan conference on security system technology, p. 67-71, sanderstead, 18-20 Oct. 1995.

[8] Priyanga .M, Raja ramanan.V, "Unmanned Aerial Vehicle for Video Surveillance Using Raspberry Pi,” International Journal of Innovative Research in Science, Engineering and Technology, Volume 3, Special Issue 3, pp, 1715-1720, March 2014

[9] D.R.Gowthaman, R.Bhalamurugan, T.Balaji, V. Manoj kuma,"Design and Modeling of Unmanned Surface Vehicle", The proceeding of 2014 international conference on computation of power, energy, information and communication (iccpeic), pp.283-289, 2104

[10] Md Saiful Islam, Uipil Chong, "Detection of Uncooperative Targets Using Cross-Correlation in Oceanic Environment", International Journal of Digital Content Technology and its Applications(JDCTA), Volume 7, Number 12, August 2013

About Author (s):

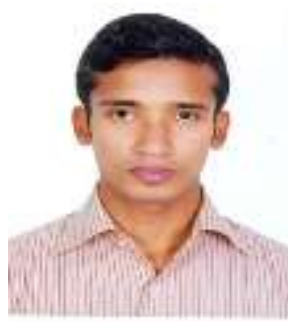

Md Saiful Islam is a lecturer of Electrical and Electronic Engineering at Premier University, Bangladesh. He received the B. S. degree in Electrical and Electronic Engineering from Chittagong University of Engineering and Technology, Bangladesh, in 2010, and M.S. degree in Electrical Engineering from University of Ulsan, Korea in 2014.

Now, he is doing his $\mathrm{PhD}$ in School of Electrical and Computer Engineering at University of Ulsan, South Korea and working as research assistant in Digital Signal processing and New Media lab(DSPNM) at the same University. He is currently researching the following advanced field: radar signal processing.

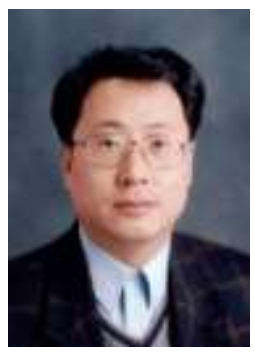

Dr. Jung-Chul Lee received the B. S., M.S. and $\mathrm{Ph}$. D. degree in Electronic Engineering from Seoul National University, Korea. He worked at ETRI for fifteen years on speech processing and speech communication systems. In August of 2002, he joined the School of Computer Engineering and Information Technology of the University of Ulsan in Ulsan City, Korea. His research interests are in digital/speech signal processing, microprocessor application with

applications to text-to-speech conversion, speech recognition, and speech coding.

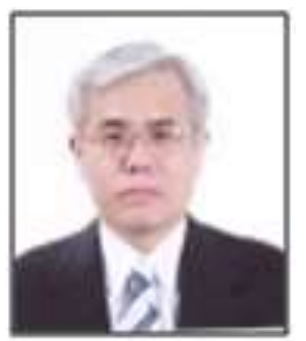

Dr. Ui-Pil Chong received the B. S. degree in Electrical Engineering from University of Ulsan, Korea, and M.S. degree in Computer Engineering from Oregon State University, USA and Ph.D.degree from New York University(POLY), USA. In January of 1997, Dr. Chong joined the School of Computer Engineering and Information Technology of the University of Ulsan in Ulsan City, Korea.

He has published more than 280 journal papers, conference papers in the area of Digital Signal Processing, Fault Detection and Diagnosis in the plants, Biomedical Engineering, Computer Music, and Multimedia Applications. He also holds the 20 Korean patents. He is a member of IEEE since 1993 and Eta Kappa Nu since 1995. Currently, He is the Head of Whale Research Institute in University of Ulsan and Vice President of the Korea Institute of Signal Processing and Systems. 